\title{
Afadin is localized at cell-cell contact sites in mesangial cells and regulates migratory polarity
}

\author{
Haruko Tsurumi ${ }^{1}$, Hidetake Kurihara ${ }^{2}$, Kenichiro Miura ${ }^{1}$, Atsushi Tanego ${ }^{1}$, Yasutaka Ohta ${ }^{3}$, Takashi Igarashi ${ }^{4}$, Akira Oka $^{1}$,
} Shigeru Horita ${ }^{5}$, Motoshi Hattori ${ }^{5}$ and Yutaka Harita ${ }^{1}$

In kidney glomeruli, mesangial cells provide structural support to counteract for expansile forces caused by pressure gradients and to regulate the blood flow. Glomerular injury results in proliferation and aberrant migration of mesangial cells, which is the pathological characteristic of mesangial proliferative glomerulonephritis. To date, molecular changes that occur in mesangial cells during glomerular injury and their association with the pathogenesis of glomerulonephritis remain largely unclear. During the search for proteins regulating the morphology of mesangial cells, we found that afadin, a multi-domain F-actin-binding protein, and $\beta$-catenin are expressed in cell-cell contact sites of cultured mesangial cells and mesangial cells in vivo. Afadin forms a protein complex with $\beta$-catenin in glomeruli and in cultured mesangial cells. Protein expression of afadin at mesangial intercellular junctions was dramatically decreased in mesangial proliferative nephritis in rats and in patients with glomerulonephritis. RNA interference-mediated depletion of afadin in cultured mesangial cells did not affect proliferation rate but resulted in delayed directional cell migration. Furthermore, reorientation of the Golgi complex at the leading edges of migrating cells in wound-healing assay was disturbed in afadindepleted cells, suggesting the role of aberrant migratory polarity in the pathogenesis of proliferative glomerulonephritis. These data shed light on glomerulonephritis-associated changes in cell-cell adhesion between mesangial cells, which might be related to migratory polarity.

Laboratory Investigation (2016) 96, 49-59; doi:10.1038/labinvest.2015.133; published online 16 November 2015

In kidney glomeruli, mesangial cells together with their surrounding mesangial matrix material constitute the mesangium that provides the supporting structure to counteract for expansile forces caused by pressure gradients across the glomerular basement membrane. ${ }^{1}$ Neighboring mesangial cells are connected by intercellular junctions, providing stability against tensional forces. Previous studies have demonstrated that $\alpha$-catenin, $\beta$-catenin, and $\mathrm{N}$-cadherin localize at cell-cell contact sites of mesangial cells in the rat kidney, suggesting that membrane-bound adherens junctions formed by cadherin-catenin system mechanically connect mesangial cells. ${ }^{2,3}$ The gap junction proteins, connexin 40 and 43 , have also been found at cell-cell contact sites in the rodent kidney mesangial cells and in cultured rat mesangial cells,, 4 suggesting the possible role of gap junctions in the formation of the functional syncytium of the mesangium.

Glomerular injury in vivo results in the phenotypical alteration of mesangial cells. In an experimental animal model of mesangial proliferative nephritis (anti-Thy-1 model), ${ }^{6,7}$ mesangial injury causes mesangiolysis that is manifested by the attenuation or dissolution of the mesangial matrix and degeneration of mesangial cells. This process is followed by reconstitution of the mesangium through coordinated migration and proliferation of mesangial cells, ${ }^{8-10}$ which is mediated by cytokines and growth factors, such as the platelet-derived growth factor (PDGF). ${ }^{11,12}$ The activation of mesangial cells induced by cytokines or growth factors is a histological hallmark of human mesangioproliferative glomerulonephritis. ${ }^{13,14}$ To date, the molecular composition of cell-cell interaction sites between human mesangial cells remains largely unclear both in the normal physiological state and in human mesangial proliferative nephritis.

During the search for proteins that regulate the morphology of mesangial cells, we found that afadin, a tumor suppressorlike protein encoded by MLLT4, was localized in cell-cell contact sites of mesangial cells in vitro and in vivo. Afadin is a

\footnotetext{
${ }^{1}$ Department of Pediatrics, Graduate School of Medicine, The University of Tokyo, Tokyo, Japan; ${ }^{2}$ Department of Anatomy, Juntendo University School of Medicine, Tokyo, Japan; ${ }^{3}$ Division of Cell Biology, Department of Biosciences, School of Science, Kitasato University, Kanagawa, Japan; ${ }^{4}$ National Center for Child Health and Development, Tokyo, Japan and ${ }^{5}$ Department of Pediatric Nephrology, Tokyo Women's Medical University, Tokyo, Japan

Correspondence: Dr Y Harita, MD, PhD, Department of Pediatrics, Graduate School of Medicine, The University of Tokyo, 7-3-1 Hongo, Bunkyo-ku, Tokyo 113-8655, Japan. E-mail: haritay-ped@h.u-tokyo.ac.jp
}

Received 16 February 2015; revised 14 August 2015; accepted 17 October 2015 
205-kDa F-actin-binding protein that contains two Rasbinding domains within the amino terminus followed by kinesin and myosin-like domains and a PDZ module in the middle of the protein. ${ }^{15}$ Afadin is expressed in epithelial cells, neurons, fibroblasts, and endothelial cells, ${ }^{16}$ and is localized in epithelial adherens junctions ${ }^{15}$ consisting of two adhesion complexes (nectin-afadin complex and E-cadherin-catenin complex $)^{17}$ and is also found in tight junctions. ${ }^{18,19}$ Afadin interacts with cell adhesion molecules, cytoskeletal components, and signaling molecules and is generally considered to function as an adaptor protein. ${ }^{20}$

In this study, we analyzed the expression of mesangial cell adhesion molecules under physiological and pathological conditions. Our results demonstrate that afadin forms a protein complex with $\beta$-catenin at cell-cell adhesion sites in mesangial cells and suggest that phenotypical alterations in mesangial cell-cell junctions might be associated with misdirected mesangial migration in proliferative glomerulonephritis.

\section{MATERIALS AND METHODS}

\section{Antibodies and Reagents}

The following antibodies were obtained from commercial sources: mouse monoclonal anti-afadin antibody (BD, Franklin Lakes, NJ, USA) for immunostaining of human and rat tissue samples; rabbit polyclonal anti-Mllt4 antibody (Sigma, St Louis, MO, USA) for immunoblotting and immunostaining of cultured human mesangial cells; rabbit polyclonal anti-l-afadin antibody (Abcam, Cambridge, MA, USA) for immunoelectron microscopy; mouse monoclonal anti- $\beta$-catenin antibody (BD); mouse monoclonal anti-ZO-1 antibody (Zymed, San Francisco, CA, USA); mouse monoclonal $\alpha$-SMA antibody (Progen, Heidelberg, Germany); rabbit monoclonal anti- $\beta$-actin antibody (Cell Signaling, Danvers, MA, USA); BODIPY FL phallacidin B607 (Life Technologies, Grand Island, NY, USA); mouse monoclonal anti-golgin-97 antibody (Invitrogen, Carlsbad, CA, USA) for immunostaining of cultured human mesangial cells. PDGF-BB was obtained from Sigma. Full-length pEGFP-l-afadin was kindly gifted from Professor Takai (Kobe University). ${ }^{21}$

\section{Cell Culture and RNAi}

Human embryonic kidney HEK293T cells (ATCC, Manassas, VA, USA) were maintained as previously described. ${ }^{19}$ Primary human mesangial cells (Lonza, Walkersville, MD, USA) were routinely cultured and maintained as previously described. ${ }^{19}$ Cells (passages 7-9) were grown in DMEM supplemented with 10\% FBS until $80 \%$ confluence was reached. Before stimulation, cells were rendered quiescent by maintaining them in serum-free medium for $24 \mathrm{~h}$. Transfections of HEK293T cells were performed using Lipofectamine 2000 reagent (Invitrogen) as per manufacturer's instructions. The sequences of small interfering RNAs (siRNAs) used are as follows: non-silencing control (QIAGEN Cat\#1027281,
Venlo, The Netherlands), afadin siRNA\#1 (5'-UGAGAAAC CUCUAGUUGUATT- $\left.3^{\prime}\right)$ and afadin siRNA\#2 (5'- GUUAAG GGCCCAAGACAUATT-3'). The siRNA duplexes were transfected into human mesangial cells using Lipofectamine RNAiMAX (Invitrogen) as per manufacturer's instructions.

\section{Western Blotting}

Cell lysates were separated by SDS-PAGE and transferred to polyvinylidene difluoride membranes. Horseradish peroxidase-conjugated anti-rabbit IgG was used as the secondary antibody (GE Healthcare, Buckinghamshire, UK). Peroxidase activity was detected using the ECL or ECL-Plus system (GE Healthcare).

\section{Co-Immunoprecipitation}

Cells were lysed in a lysis buffer (20 mM Tris- $\mathrm{HCl}(\mathrm{pH} 7.5)$, $150 \mathrm{mM} \mathrm{NaCl}, 1 \mathrm{mM}$ EDTA, and 1\% NP-40) containing a protease inhibitor cocktail (Roche, Germany) for $15 \mathrm{~min}$ on ice. Lysates were clarified by centrifugation and incubated with agarose beads conjugated with 9E10 anti-Myc antibody for $1 \mathrm{~h}$ at $4{ }^{\circ} \mathrm{C}$. For immunoprecipitation of endogenous proteins, rabbit IgG or rabbit anti-afadin IgG was incubated with protein $\mathrm{G}$ beads for $1 \mathrm{~h}$ at $4{ }^{\circ} \mathrm{C}$ followed by incubation with cell lysates for $1 \mathrm{~h}$ at $4{ }^{\circ} \mathrm{C}$. Beads were washed twice with Tris-buffered saline-1\% NP-40, and bound proteins were eluted with $100 \mathrm{mM}$ glycine- $\mathrm{HCl}(\mathrm{pH}$ 2.6). Proteins were resolved by SDS-PAGE for immunoblot analysis.

\section{Immunohistological Analysis}

Samples from rat kidneys were perfused with PBS, embedded in the OCT compound, and frozen. Cryosections (3-5- $\mu \mathrm{m}$ thickness) were then cut using a Jung Frigocut 2800E (Leica, Wetzlar, Germany) and mounted on silanecoated glass slides. The sections were fixed in acetone for $1 \mathrm{~min}$, incubated with primary antibodies, and stained with Alexa Fluor-conjugated secondary antibodies (Invitrogen). Samples were observed on an inverted microscope (model IX71; Olympus, Tokyo, Japan) and images were obtained using a cooled charge-coupled device camera (ORCA-ER; Hamamatsu Photonics, Shizuoka, Japan) controlled by Aqua-Lite software (Hamamatsu Photonics). Confocal fluorescent images (Figure $2 \mathrm{a}$ and $\mathrm{c}$ ) were obtained by a Zeiss LSM 700 confocal microscope. The images were processed using Adobe Photoshop CS5.

\section{Immunoelectron Microscopy}

Kidney specimens derived from a normal human control were cut into ultrathin cryosections. Sections were transferred to nickel grids (150 mesh) that had been coated with Formver and carbon. Subsequent incubation steps were performed by floating grids on droplets of the filtered solution. After quenching free aldehyde groups with PBS-0.01 M glycine, sections were incubated overnight with primary antibodies and then incubated with secondary antibodies coupled to 10-nm gold particles (diluted 1:100 with PBS containing 10\% 
fetal calf serum) for $1 \mathrm{~h}$. After immunostaining, sections were fixed with $2.5 \%$ glutaraldehyde buffered with $0.1 \mathrm{M} \mathrm{PB}$ ( $\mathrm{pH}$ 7.4). Sections were then contrasted with $2 \%$ neutral uranyl acetate solution for $30 \mathrm{~min}$, absorption-stained with $3 \%$ polyvinyl alcohol containing $0.2 \%$ acidic uranyl acetate for $30 \mathrm{~min}$, and observed with a JEM1230 transmission electron microscope (JEOL, Tokyo, Japan).

\section{Patients}

Formalin-fixed, paraffin-embedded tissue samples were obtained by kidney biopsies from seven patients admitted to the Kidney Center, Tokyo Women's Medical University. IgA nephropathy patient 1 (IgAN 1) presented with hematuria and proteinuria at 8 years of age. She was diagnosed with IgAN on a kidney biopsy at age 10. IgAN 2 was 6 years of age when he had an episode of macrohematuria followed by moderate hematuria and proteinuria. One month later, a kidney biopsy was performed and IgAN was diagnosed. IgAN 3 presented with macrohematuria followed by moderate hematuria and proteinuria at 9 years of age. IgAN 4 presented with hematuria and proteinuria at 7 years of age when she was diagnosed with IgAN. The second biopsy at 12 years of age was used in this article. The sample from IgAN 5 was obtained at 5 years of age when he had moderate hematuria and proteinuria. Membranoproliferative gromerulonephritis patient 1 (MPGN 1) presented with moderate hematuria and proteinuria, and diagnosed as MPGN on a kidney biopsy at 7 years of age. MPGN 2 presented with moderate hematuria and was diagnosed as MPGN on a first kidney biopsy at 10 years of age. The second biopsy at 12 years of age was used in this article. Normal tissue samples (adult donor kidneys) were used as controls. This study was approved by the ethics committee of the Tokyo Women's Medical University.

\section{Proximity Ligation Assay}

Proximity ligation assay (PLA) was performed using the Duolink in situ PLA kit (Olink Bioscience, Uppsala, Sweden). Human mesangial cells were cultured on coverslips coated with collagen type 1 and fixed, and permeabilized. After washing in phosphate-buffered saline, cells were blocked in the blocking solution at $37^{\circ} \mathrm{C}$ for $30 \mathrm{~min}$. After washing, samples were incubated with diluted primary antibodies (anti-Mllt4 antibody (HPA049868) and anti- $\beta$-catenin antibody (BD51-9001921)) followed by incubation with corresponding secondary antibodies conjugated to PLA oligonucleotide probes (Duolink PLA Rabbit PLUS and PLA Mouse MINUS proximity probes) for $2 \mathrm{~h}$ at $37^{\circ} \mathrm{C}$. Hybridization and ligation of connector oligonucleotides, rolling-circle amplification, and detection of amplified DNA products using detection reagent Red were performed using the Duolink detection reagent kit according to the manufacturer's protocol. The samples were co-stained with BODIPY FL phallacidin (green) and DAPI (4',6-diamidino-2phenylindole, blue) before mounting.

\section{Induction of Thy-1.1 Nephritis}

All procedures performed on laboratory animals were approved by the Institutional Animal Care Committee of the University of Tokyo and were conducted in compliance with guidelines for animal experimentation of the University of Tokyo. Six-week-old male Wistar rats were obtained from Charles River Japan (Kanagawa, Japan). Animals were kept in an air-conditioned room and given free access to standard rat chow and tap water. To induce Thy-1.1 nephritis, the mouse monoclonal anti-Thy-1.1 antibody E30 (200 $\mu$ g per rat) was intravenously injected into rats under anesthesia with diethyl ether. ${ }^{22}$ Kidneys from control and Thy-1.1 nephritis rats were obtained at days $0,1,5,8$ and 14 after the antibody injection and used for immunoblotting and immunohistochemical analyses.

\section{Wound-Healing Assay}

Human mesangial cells $\left(5 \times 10^{4}\right)$ transfected with control siRNA or afadin siRNA \#1 or \#2 were cultured on collagencoated cover slips in 24-well plates for 3 days prior to wounding with a pipette tip. Phase-contrast images were acquired at 0,16 , and $24 \mathrm{~h}$ after wounding. The wound area (nine parts per cover slip) was determined using ImageJ software. The assays were performed in triplicates.

\section{Golgi Apparatus Polarization Measurements}

Golgi apparatus polarization was measured using a previously described method. ${ }^{23}$ To record the position of the Golgi apparatus in migrating wound-edge cells, wounds were made in monolayers of human mesangial cells using a yellow pipette tip and cells were fixed $4 \mathrm{~h}$ after wounding. The cells were immunostained using mouse monoclonal anti-golgin-97 antibody and DAPI. To determine the orientation of the Golgi apparatus, $120^{\circ}$ angles were drawn from the center of the nuclei of cells that lined the edge of the wound, creating three sectors: one facing the wound and two faces away from the wound. Golgi were classified as facing the wound (those in the sector facing the wound), facing another direction (those in either sector facing away from the wound), or non-polarized (those in two or more sectors). The assays were performed in triplicates.

\section{Boyden Chamber Assay}

Cell migration was assayed in Boyden chambers with $8-\mu \mathrm{m}$ pore size Fluoroblok 24-well inserts (BD). The membranes are manufactured with a biologically inert coating that blocks the transmission of visible light at wavelengths of $490-700 \mu \mathrm{m}$, allowing selective visualization of fluorescently labeled cells at the bottom of the chamber. Human mesangial cells transfected with control siRNA or afadin siRNA \#1 or \#2 were cultured in serum-free DMEM containing $0.2 \%$ FCS for $24 \mathrm{~h}$ and then trypsinized and counted. Cells $\left(5 \times 10^{4}\right)$ in DMEM containing $0.2 \%$ FBS $(0.4 \mathrm{ml})$ were added to the upper chamber and $0.9 \mathrm{ml}$ of the same medium with or without $30 \mathrm{ng} / \mathrm{ml}$ PDGF was added to the lower chamber. 
a

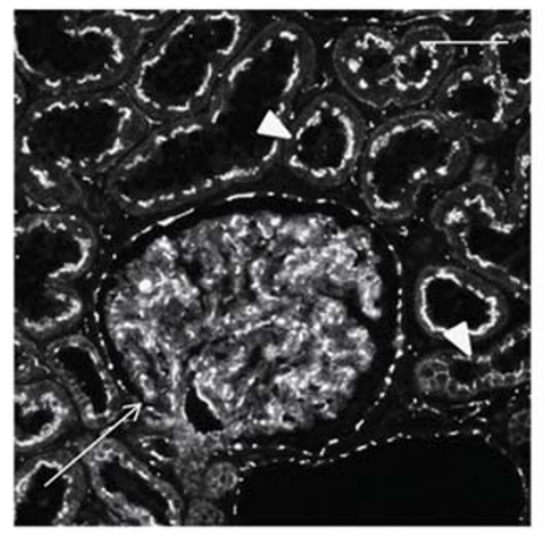

b
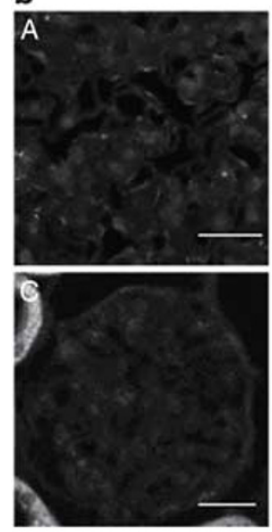
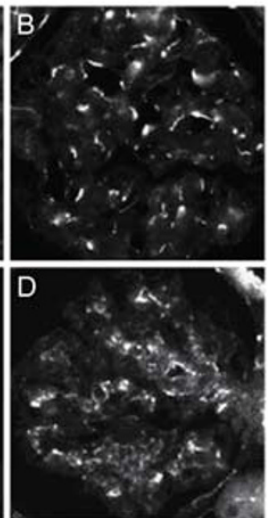

c

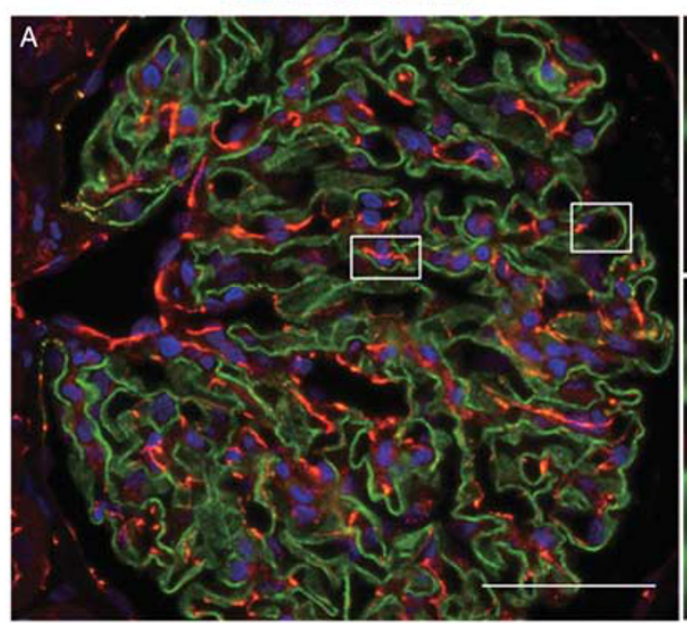

afadin-ZO-1-DAPI

d

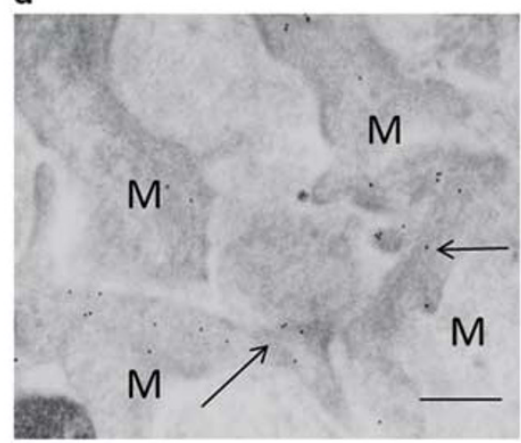

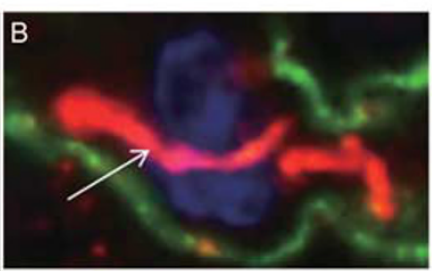
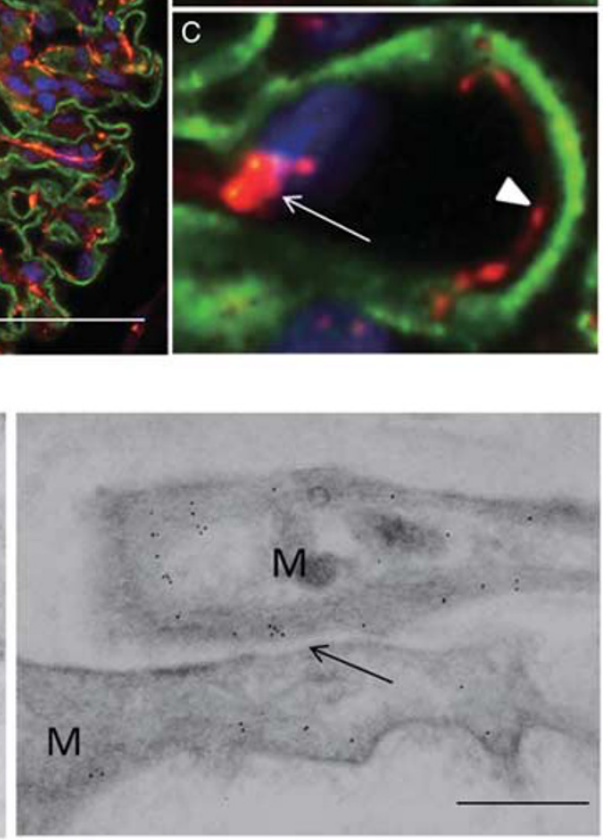

Figure 1 Expression of afadin in the kidney cortex. (a) Sections of human kidneys were stained with anti-afadin antibody. Afadin signals were detected in glomeruli (arrow), and apical side of tubular epithelial cells (arrowheads). Scale bar, $50 \mu \mathrm{m}$. (b) Sections of human (A and B) and rat (C and D) kidney were stained with anti-afadin antibody preadsorbed with anti-myc immunoprecipitates from cell lysates of HEK293 cells expressing myc-tagged l-afadin ( $A$ and $C$ ) or control vector (B and D). (c) Dual-labeling immunofluorescence of afadin (red) and ZO-1 (green) in the human kidney. Strong afadin signals were detected in the mesangium, particularly in cell-cell adhesions between mesangial cells ( $B$, arrow) and between mesangial and endothelial cells (C, arrow). Afadin signals were also detected at cell-cell adhesions between endothelial cells of glomerular capillaries (C, arrowhead). Highermagnification images corresponding to squares in A are shown in B and C. Scale bar, $50 \mu \mathrm{m}$. (d) Immunogold staining of afadin detected using immunoelectron microscopy. Immunogold particles specific for afadin were detected in the mesangial cell-cell adhesion sites (arrows). Scale bars, $200 \mathrm{~nm}$. M, mesangial cell.

Transwells were incubated for 0,2 , 4 , or $6 \mathrm{~h}$ at $37^{\circ} \mathrm{C}$. Cells were then stained with BODIPY FL phallacidin and DAPI. Phallacidin- and DAPI-positive cells on the underside of the insert in four randomly chosen fields per filter were counted under a microscope at a magnification of $\times 100$. The assays were performed in triplicates.

\section{RESULTS}

\section{Afadin Expression in the Kidney Cortex}

In the search of cell adhesion molecules in cell-cell junctions of mesangial cells, we analyzed the expression of several molecules that are known to have important roles in the formation of adherens junctions. Among them, we found that afadin was expressed in mesangial cells. Immunohistochemical staining of adult human kidney tissues revealed that afadin protein was expressed in glomerular cells, tubular epithelial cells, and extraglomerular vascular endothelial cells
(Figure 1a). A similar staining pattern was observed in rat kidney sections (Figure 1b).

To determine the localization of afadin in glomeruli, paraffin-embedded sections of the adult human kidney were double-labeled with zonula occludens-1 (ZO-1), a podocyte marker. Afadin was highly expressed in mesangial cells in the glomeruli (Figure 1c), particularly at cell-cell adhesion sites between mesangial cells or between mesangial and endothelial cells (Figure $1 \mathrm{cB}, \mathrm{C}$, arrows). Afadin was also detected at endothelial cell-cell junctions of glomerular capillaries (Figure 1cC, arrowhead). Afadin were also partially and weakly co-localized with ZO-1 (Figure 1cA). The localization of afadin was examined in more detail using immunoelectron microscopy (Figure 1d). Immunogold particles for afadin were detected in mesangial cells, particularly at cell-cell adhesion sites. 


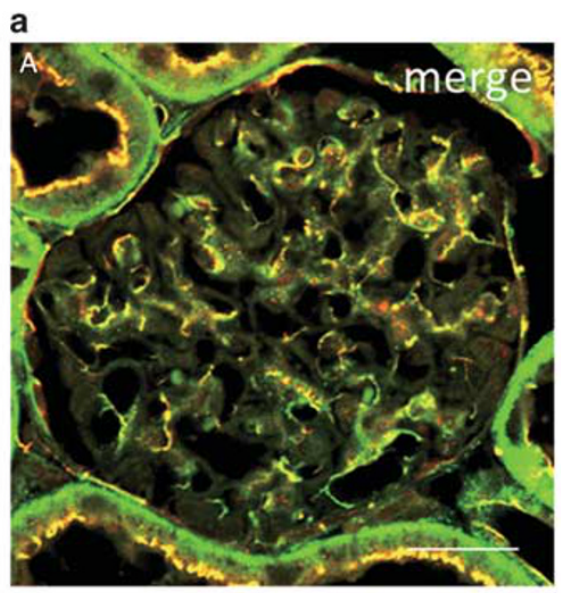

c
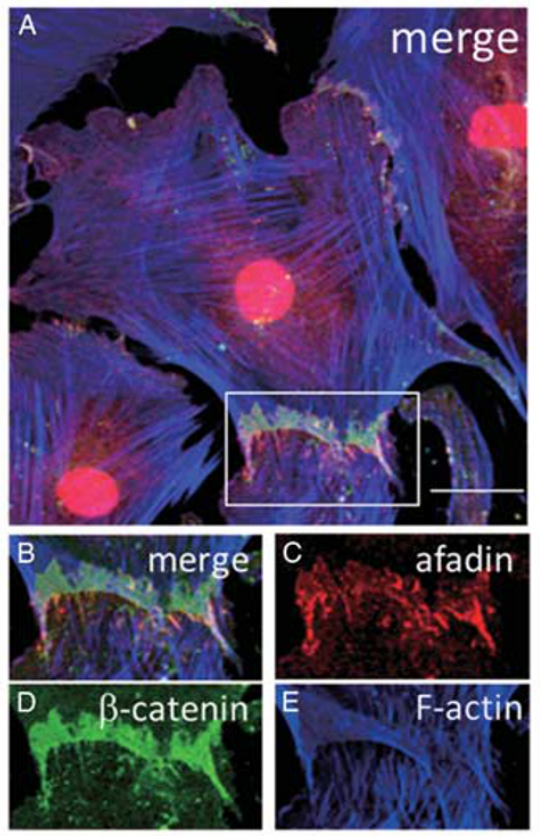

d

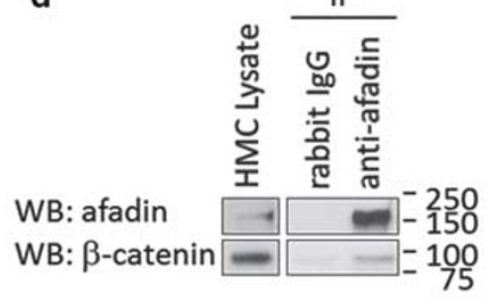

e

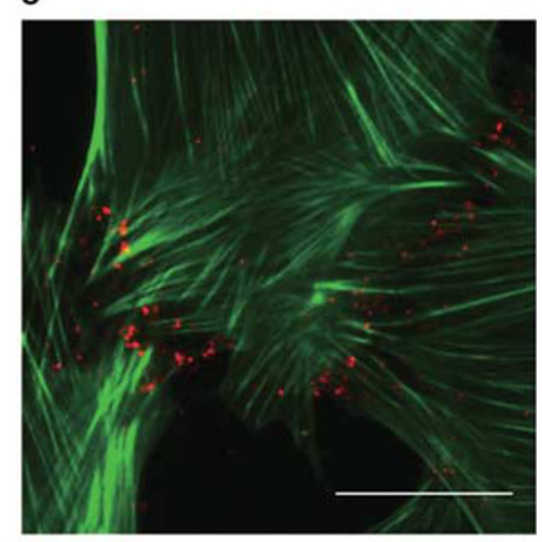

b

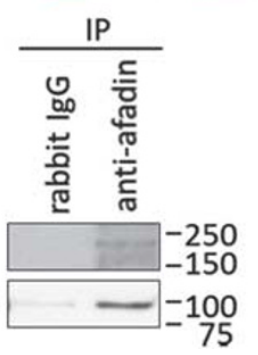

Figure 2 Association of afadin and $\beta$-catenin at mesangial cell-cell contact sites. (a) Dual-labeling immunofluorescence of afadin and $\beta$-catenin in a rat kidney. A rat kidney section was co-stained for afadin (B, red) and $\beta$-catenin (C, green). The merged image is shown in A. Scale bar, $50 \mu \mathrm{m}$. (b) Lysates of rat glomeruli were immunoprecipitated with rabbit lgG or rabbit anti-afadin antibody and were analyzed by western blotting with indicated antibodies. (c) The merged image of cultured human mesangial cells co-stained with afadin (red), $\beta$-catenin (green), and F-actin (blue). Higher-magnification images corresponding to squares in A are shown in B-E. Scale bar, $20 \mu \mathrm{m}$. (d) Lysates of cultured human mesangial cells were immunoprecipitated with rabbit IgG or rabbit anti-afadin antibody and were analyzed by western blotting with indicated antibodies. (e) In situ proximity ligation assay (PLA) in cultured human mesangial cells. Rabbit anti-afadin and mouse anti- $\beta$-catenin antibodies were combined with secondary PLA probes (Olink Bioscience). The sample was co-stained with BODIPY FL phallacidin (green). The interaction events are visible as red dots at cell-cell contact sites between mesangial cells. Scale bar, $20 \mu \mathrm{m}$.

\section{Afadin Associates With $\beta$-Catenin at Cell-Cell Contact Sites in Mesangial Cells}

Because afadin is a protein that connects membrane proteins to actin filaments (F-actin) at cell-cell junctions in epithelial cells, we searched for an afadin-binding partner in mesangial cells. Of the adhesion molecules and cytoskeletal proteins tested, we found that $\beta$-catenin, a critical component of adherens junctions, co-localized with afadin in glomeruli in vivo (Figure 2aA-C). We performed coimmunoprecipitations to investigate whether afadin interacts with $\beta$-catenin in glomeruli. As shown in Figure $2 \mathrm{~b}$, we confirmed the endogenous afadin- $\beta$-catenin interaction in isolated rat glomeruli.

Next, we examined the localization of afadin in cultured human mesangial cells. To confirm the specificity of the anti-afadin antibody, we analyzed the effects of siRNA-mediated knockdown of afadin in cultured mesangial cells. Treatment with two different siRNAs effectively decreased afadin expression at cell-cell contact sites (Supplementary Figure S1a). In cultured mesangial cells, intense signals for afadin were observed at cell-cell adhesion sites of neighboring mesangial cells, forming zipper-like structures connected to the actin cytoskeleton (Figure $2 \mathrm{cA}-\mathrm{E}$ ). Signals for $\beta$-catenin were also observed at cell-cell junctions and co-localized with those of afadin. The interaction of endogenous afadin and $\beta$-catenin in cultured mesangial cells was confirmed by immunoprecipitation analysis (Figure $2 \mathrm{~d}$ ).

To further verify the afadin- $\beta$-catenin interaction, we performed in situ PLA in cultured human mesangial cells. In situ PLA technology generates localized, discrete signals where 
a
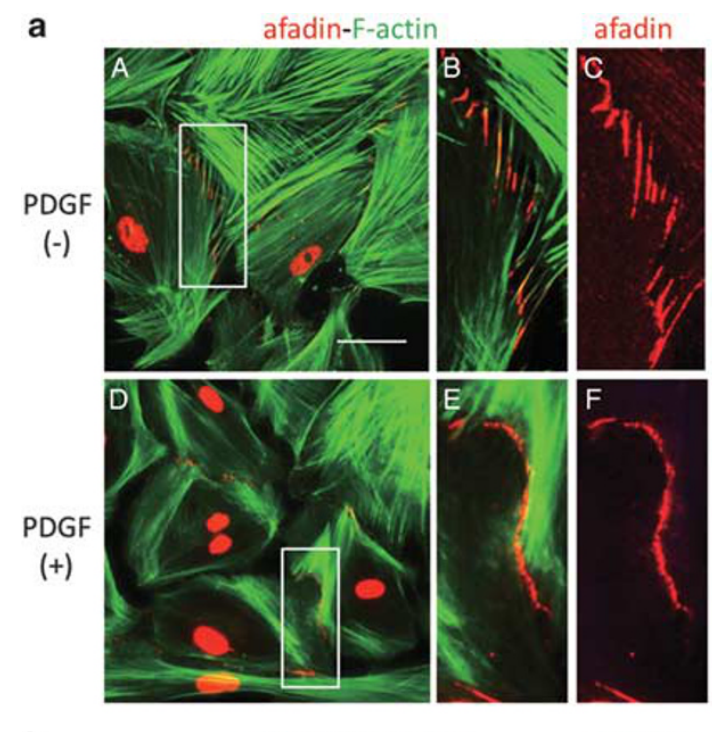

b
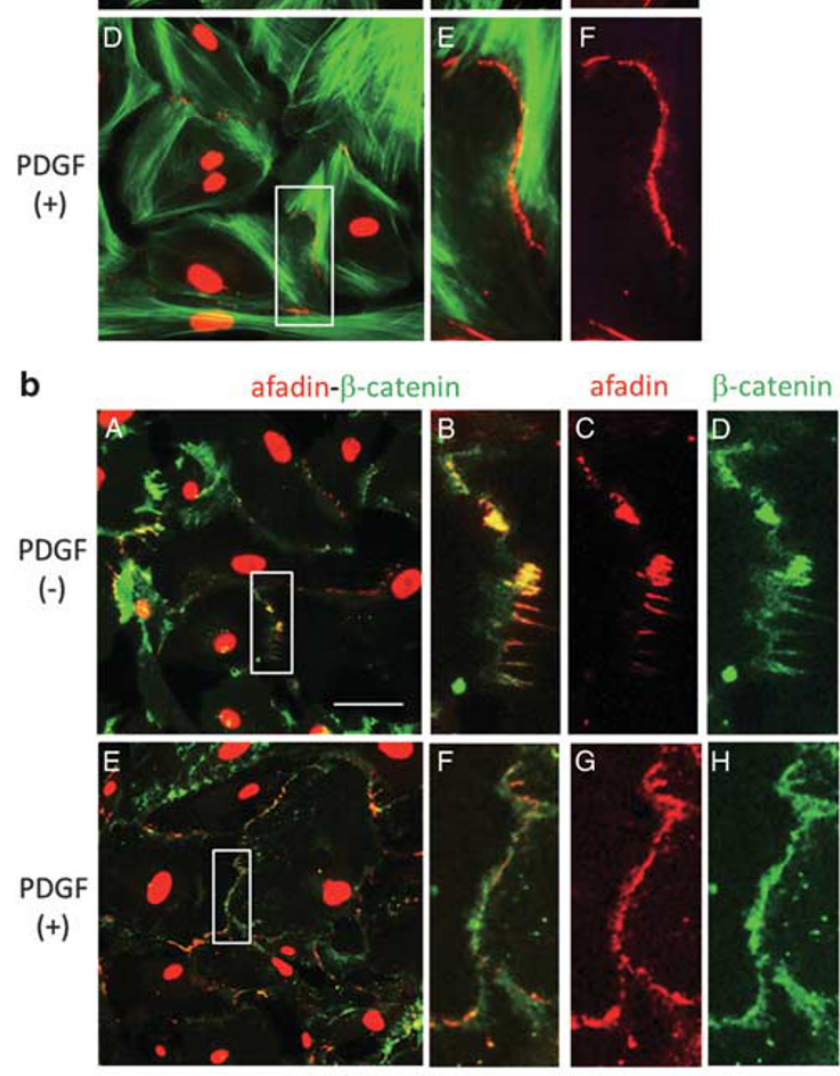

c

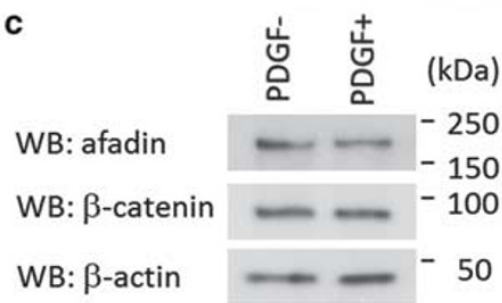

Figure 3 Alteration of the mesangial intercellular junction by PDGF. (a) The merged images of serum-starved mesangial cells stimulated with DMSO (A-C) or PDGF $(50 \mathrm{ng} / \mathrm{ml})$ for $30 \mathrm{~min}(\mathrm{D}-\mathrm{F})$, and co-stained for afadin (red) and phallacidin (green). Higher-magnification images corresponding to squares in $A$ and $D$ are shown in $B$ and $C$, and $E$ and $F$, respectively. Scale bar, $50 \mu \mathrm{m}$. (b) The merged images of serum-starved mesangial cells stimulated with DMSO (A-D) or PDGF $(50 \mathrm{ng} / \mathrm{ml})$ for $30 \mathrm{~min}(\mathrm{E}-\mathrm{H})$ co-stained for afadin (red) and $\beta$-catenin (green). Highermagnification images corresponding to squares in $A$ and $E$ are shown in B-D and F-H, respectively. Scale bar, $50 \mu \mathrm{m}$. (c) Western blot analysis of the indicated antibodies in mesangal cell lysates stimulated with or without PDGF (50 ng/ml). PDGF, platelet-derived growth factor. two proteins of interest (afadin and $\beta$-catenin, in this case) are in close proximity. As shown in Figure 2e, the afadin- $\beta$-catenin complex formation was detected as red dots, particularly at the cell-cell adhesion sites between neighboring mesangial cells.

The effect of afadin depletion on actin cytoskeleton and expression of $\beta$-catenin was analyzed. As shown in Supplementary Figure S1a, actin stress fiber organization was not significantly altered by afadin knockdown. Expression of $\beta$-catenin in cytoplasm and cell-cell contact was also not significantly altered by afadin knockdown (Supplementary Figure S1b, c).

To examine changes in cell-cell adhesion of human mesangial cells in response to a growth factor, serumstarved mesangial cells were stimulated with PDGF and then immunostained with anti-afadin antibody. When quiescent, mesangial cells possess abundant actin stress fiber structures. After PDGF treatment, the number of actin bundles in perinuclear regions decreased, and lamellipodia formation was induced at the cell periphery (Figure 3a). Zipper-like afadin expression (in red) at cell-cell contact sites was concomitantly altered to linear structures between neighboring mesangial cells. PDGF-treated mesangial cells showed similar changes in $\beta$-catenin localization at cell-cell contact sites (Figure $3 \mathrm{~b}$ ). The levels of afadin and $\beta$-catenin protein expression did not change in PDGF-treated mesangial cells (Figure $3 \mathrm{c}$ ), suggesting that intracellular localization and not the amount of afadin and $\beta$-catenin protein was altered by PDGF stimulation. These data demonstrate that the mesangial cell-cell junction and actin cytoskeleton are tightly associated under unstimulated conditions; these structures are synchronously influenced by the growth factor stimulation.

\section{Decreased Expression of Afadin in Mesangial \\ Proliferative Nephritis}

We next analyzed changes in mesangial cell-cell contact sites in proliferative nephritis in rats and humans. In rat Thy- 1 nephritis, the glomerular mesangium is destroyed by an antibody-mediated reaction followed by tissue repair mediated by migrating and proliferating mesangial cells. Before anti-Thy-1.1 injection (day 0), afadin protein was expressed in the mesangial area (Figure 4a). Alpha smooth muscle actin ( $\alpha$-SMA), a marker for activated mesangial cells, was barely detected in glomeruli at this point. At five days after the antibody injection, when prominent proliferation of mesangial cells and enlargement of the mesangium were observed, $\alpha$-SMA staining in the mesangial area became apparent and immunofluorescence signals for afadin markedly decreased (Figure 4a). The decreased afadin expression during the acute phase in the model was confirmed by western blotting of glomerular lysates (Figure $4 \mathrm{~b}$ and c). To evaluate afadin expression in human mesangial proliferative nephritis, we performed immunohistochemical analysis using kidney biopsy samples from five patients with IgAN and two patients with MPGN. These seven patients presented with 


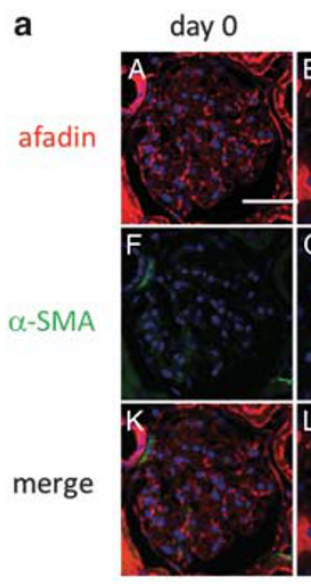

day 1
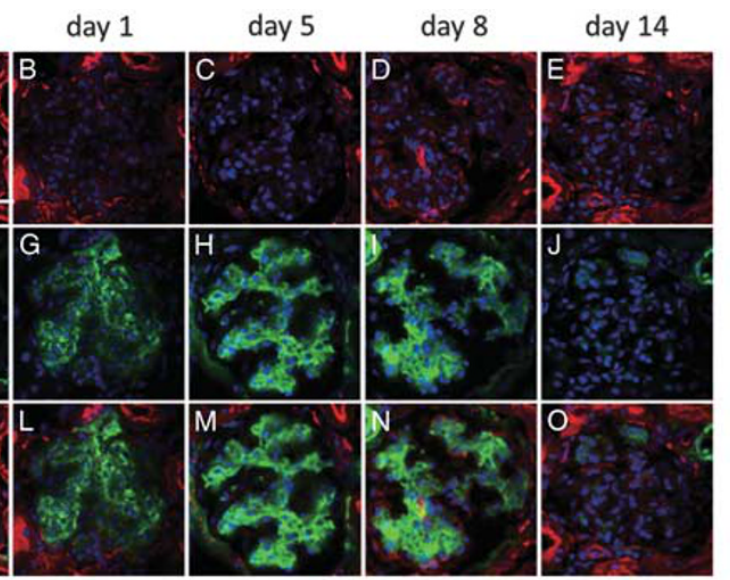

d
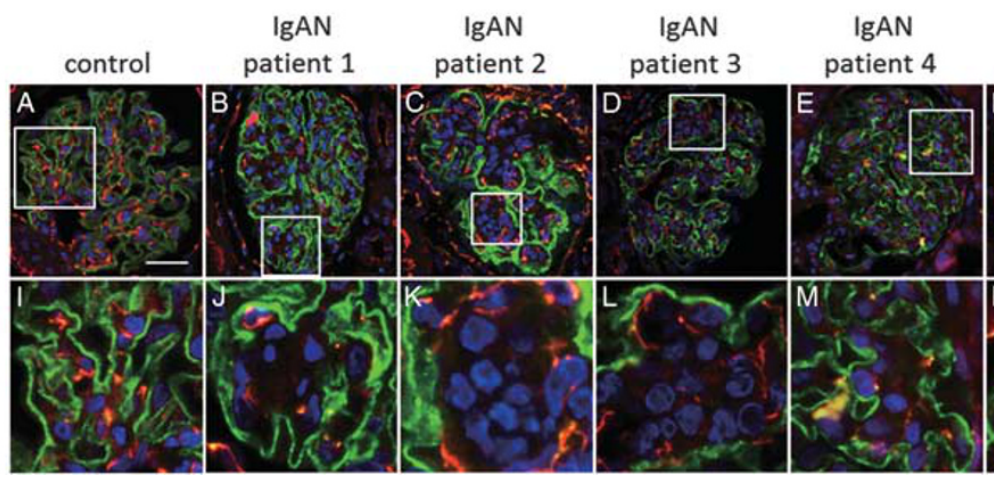

Figure 4 Decreased expression of afadin in mesangial proliferative glomerulonephritis. (a) Expression of afadin (A-E) and a-smooth muscle actin (a-SMA) (F-J) before and 1, 5, 8, and 14 days after induction of rat anti-Thy-1 glomerulonephritis. The merged images are shown in K-O. Scale bar, $50 \mu \mathrm{m}$. (b) Western blot analysis of afadin in anti-Thy-1 glomerulonephritis. Lysates of glomeruli isolated from rats before and 5, 8 and 14 days after anti-Thy-1 antibody injection were immunoblotted with indicated antibodies. The quantification of afadin in Thy- 1 nephritis was shown in c. Data are shown as the mean \pm s.d. of three independent experiments. Statistical analysis was carried out using Student $t$-test. ${ }^{*} P<0.005$. (d) Afadin expression in human glomerulonephritis. Double immunostaining for afadin (red) and zonula occludens-1 (ZO-1, green) in a control case (A) and patients with IgA nephropathy (B-F) and membranoproliferative glomerulonephritis (MPGN) (G and H) are shown. Higher-magnification images corresponding to squares in $\mathrm{A}$ through $\mathrm{H}$ are shown in I through $\mathrm{P}$, respectively. Scale bar, $50 \mu \mathrm{m}$.

moderate hematuria and proteinuria at the time of renal biopsy. As shown in Figure 4d, afadin expression in the glomerular tufts of proliferating mesangial cells especially at mesangial cellular junctions segmentally decreased. These results indicate that afadin expression is negatively regulated during the dynamic mesangial proliferative process in rodents and humans in vivo.

\section{Impaired Formation of Front-Rear Polarity by Afadin Depletion in Mesangial Cells}

To form and maintain the unique and complex three-dimensional structure of the glomerulus, intracellular organelles of mesangial cells are organized in a polarized manner. Afadin is involved in the reorientation of the Golgi apparatus in NIH3T3 cells. ${ }^{24}$ On the basis of these data, we examined whether the afadin depletion observed in proliferative nephritis in vivo is associated with the formation of front-rear polarity in mesangial cells. To this end, we performed a wound-healing assay by scratching a confluent monolayer of mesangial cells and analyzed the effect of siRNA-mediated knockdown of afadin on the alignment of the Golgi complex in cells at the wound edge. The fraction of Golgi complexes facing the wound was significantly lower in afadin knockdown than that in wild-type cells (Figure 5a and $b$ ), suggesting that afadin is required for the formation of front-rear polarity in migrating mesangial cells.

\section{Impairment of Directional Mesangial Cell Migration by Afadin Depletion}

The reorientation of the Golgi complex in moving cells correlates with the directional cell movement. ${ }^{25}$ Having identified that afadin expression was lower in mesangial proliferative nephritis and that afadin depletion results in defects in reorientation of the Golgi apparatus, we next analyzed the effect of siRNA-mediated knockdown of afadin in directional cell migration. In wound-healing assays (Figure $6 \mathrm{a}$ and $\mathrm{b}$ ), cells transfected with control siRNA moved into the wound area, gradually filling the gap created by the 
a

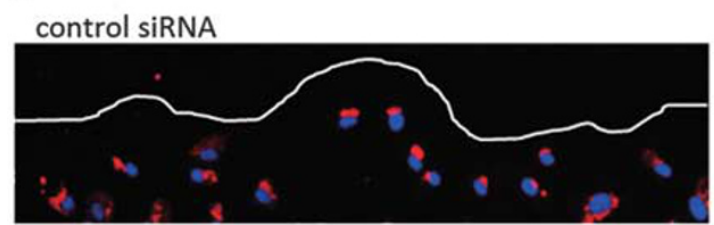

afadin SiRNA\#1

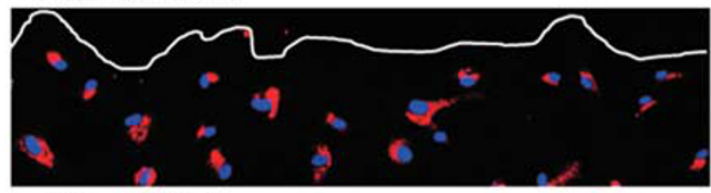

afadin siRNA\#2

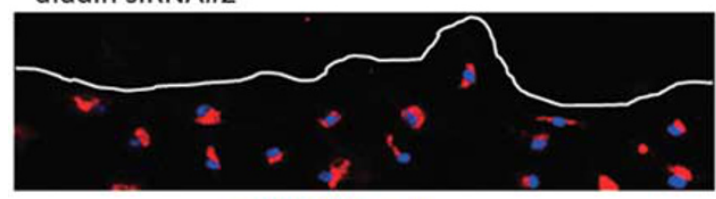

golgin97-DAPI b

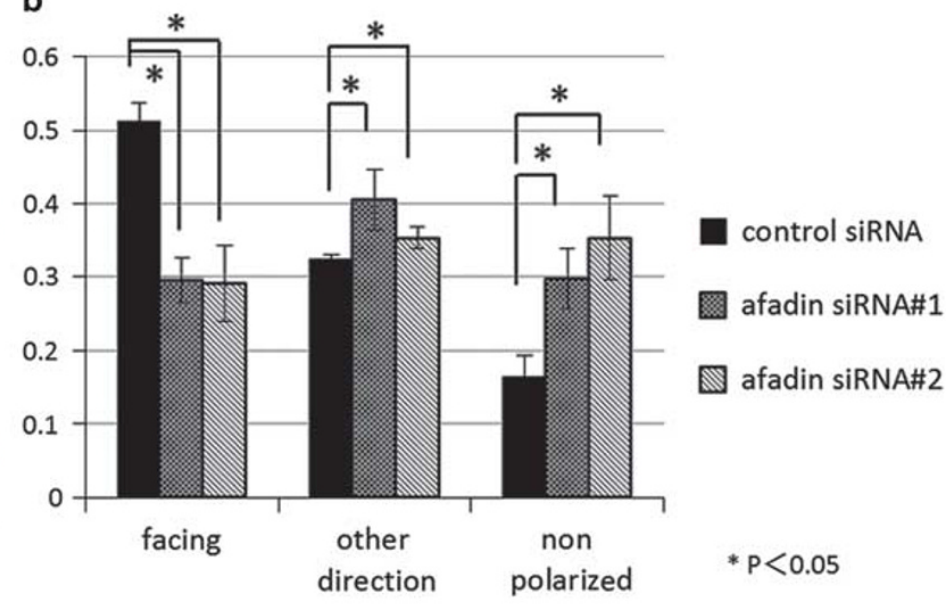

Figure 5 Impaired formation of front-rear polarity in afadin-depleted mesangial cells. (a) Confluent human mesangial cell monolayers of control siRNA and siRNAs \#1 and \#2 for afadin were manually scratched and cultured for $24 \mathrm{~h}$. Cells were stained with goldin97 (golgi complex marker, red) and DAPI (blue). The line indicates the leading edge of the wound. (b) The percentage of Golgi apparatus facing the wound, facing the other direction, and nonpolarized with respect to the wound was calculated as described in the Materials and Methods. ${ }^{*} P<0.05$ using Student's $t$-test. Data are shown as the mean \pm s.d. of three independent experiments.

scratch. In contrast, cells treated with siRNA against afadin failed to close the gap in the same time period. We examined the effect of afadin depletion in cell proliferation during the experimental period $(24 \mathrm{~h})$. BrdU cell proliferation assay revealed that the velocity of cell proliferation in wild-type and afadin-knockdown cells were not significantly different (data not shown). Next we performed a modified Boyden chamber assay to examine the effect of afadin knockdown in PDGF-mediated directed migration (Figure 6c). Treatment with two distinct siRNAs targeting afadin significantly delayed cell migration in response to PDGF (Figure 6c), suggesting afadin has a role in the migratory polarity of mesangial cells.

\section{DISCUSSION}

Mesangial cells contain convoluted F-actin fibers that are connected to the mesangial extracellular matrix (ECM), glomerular basement membrane, and cell-cell adhesion sites between mesangial cells or between mesangial and endothelial cells. ${ }^{26}$ We demonstrated here that cell-cell contact sites of mesangial cells are formed by an afadin- $\beta$-catenin protein complex, and this structure might regulate the spatial organization of cells in the glomerulus under physiological and pathological conditions by connecting cell-cell adhesion molecules to cytoskeletal structures.

Afadin is an actin-filament-binding protein that binds to nectin, an immunoglobulin-like cell-cell adhesion molecule, in epithelial cells. ${ }^{27,28}$ Afadin also associates with $\alpha$-catenin through ponsin-vinculin and afadin DIL-domain-interacting protein- $\alpha$-actinin units. ${ }^{16} \alpha$-catenin associates with $\beta$-catenin, which is recognized by the cytoplasmic domain of cadherins. Through these interactions, afadin can recruit various membrane proteins, resulting in the formation of adherens junctions. ${ }^{27}$ Afadin is also expressed in non-epithelial cells, such as NIH3T3 cells, and is localized at the leading edge of moving cells, regulating directional cell movement in cooperation with the PDGF receptor or Rap1. ${ }^{24}$ Because calcium depletion did not affect afadin/catenin localization in cultured human mesangial cells (data not shown), $\mathrm{Ca}^{2+}$-independent mesangial cell-cell interactions should exist. At present, the extracellular structure of the mesangial cell-cell junction is not known either in vivo or in vitro. We could not detect signals for nectin-3 or nectin-4, N-cadherin, or connexin $40 / 43$ at the cell-cell contact sites of human cultured mesangial cells or mesangial cells in human kidney specimens (data not shown). However, as afadin and $\beta$-catenin bind to both transmembrane proteins and actin, binding between the adhesion complex and cytoskeleton during the junctional development and dynamic remodeling must be coordinated by these adaptor proteins at cell-cell contact sites in mesangial cells.

Mesangial cells undergo phenotypical changes in glomerulonephritis. Under normal conditions, mesangial cells express non-muscle isoforms of actin, namely $\beta$ - and $\gamma$-cytoplasmic actin, in vivo. ${ }^{29}$ In human and experimental rat glomerulonephritis, mesangial cells acquire myofibroblastlike characteristics expressing smooth muscle cell-type actin. ${ }^{29}$ In these activated mesangial cells, afadin expression was downregulated. As we previously demonstrated, EPLIN, 
a

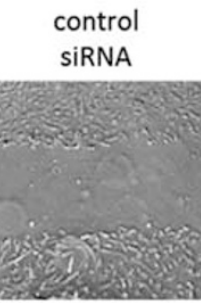

$24 \mathrm{~h}$
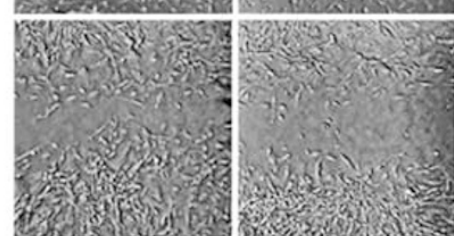

PDGF -

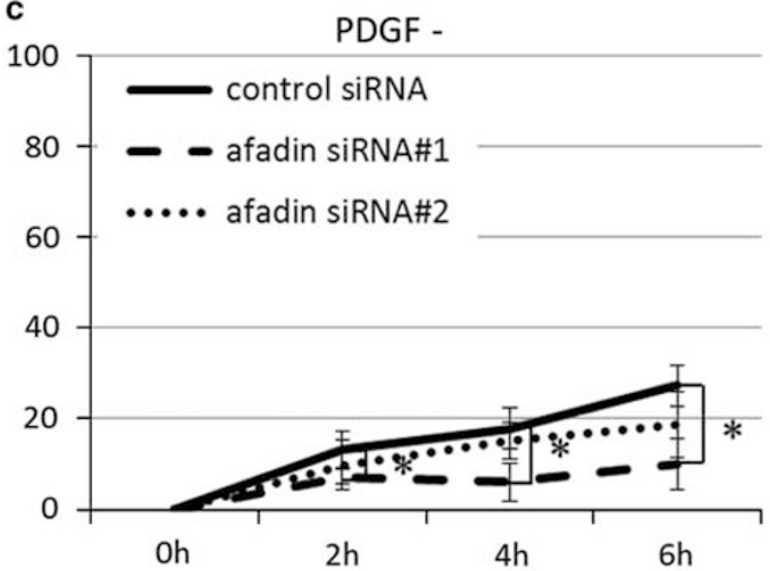

b

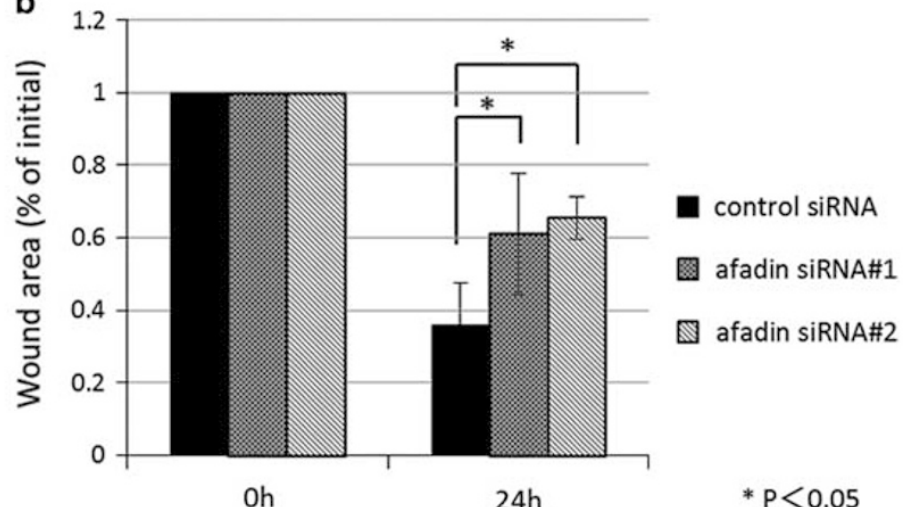

$\mathrm{Oh}$

$24 \mathrm{~h}$

$* \mathrm{P}<0.05$

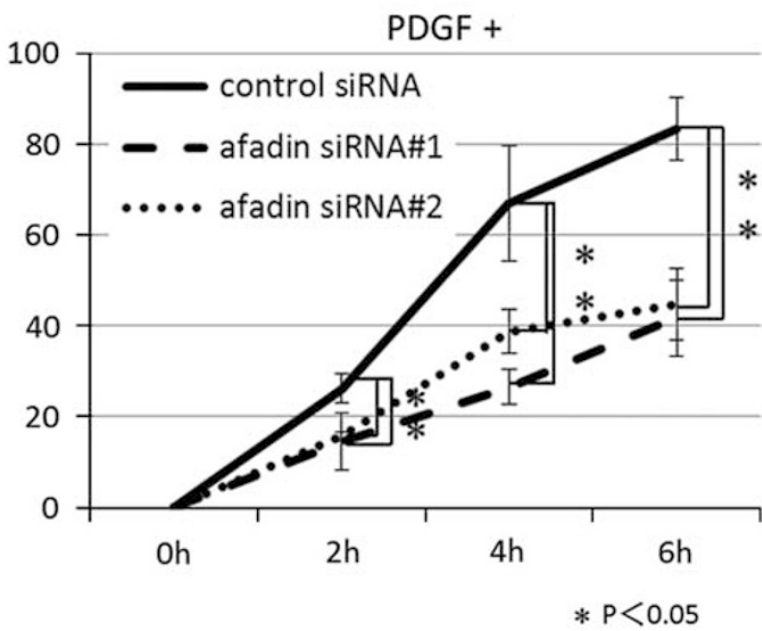

Figure 6 Impairment of directional mesangial cell migration by afadin depletion. (a) Confluent mesangial cell monolayers were manually scratched and cultured for $24 \mathrm{~h}$. Light-microscopy images of confluent human mesangial cell monolayers of control siRNA and siRNAs \#1 and \#2 for afadin immediately after wounding $(0 \mathrm{~h})$ and $24 \mathrm{~h}$ later. (b) The percent of initial wound area was calculated as described in the Materials and Methods.

$P<0.05$ using Student's $t$-test. Data are shown as the mean \pm s.d. of three independent experiments. (c) Three days after transfection with control siRNA or afadin siRNAs, mesangial cells were subjected to a modified Boyden chamber assay with or without PDGF $(30 \mathrm{ng} / \mathrm{ml})$ in the lower chamber. ${ }^{*} P<0.05$ using Student's $t$-test. Data are shown as the mean \pm s.d. of three independent experiments.

an actin-binding protein that crosslinks actin filaments, is also downregulated in mesangial proliferative nephritis in rodents and humans. ${ }^{30}$ On the other hand, the expression of other actin-binding proteins, such as profilin and drebrin, is upregulated in proliferative mesangial cells. ${ }^{31-33}$ The simultaneous induction or suppression of clusters of actin cytoskeleton regulatory genes or cell adhesion proteins has been reported in stimulated cultured mesangial cells. ${ }^{32}$ Our data demonstrate that changes in cell-cell adhesion and the cytoskeleton concurrently occur in proliferating mesangial cells in vivo.

There are probably several mechanisms by which changes in afadin expression affect mesangial cell function. First, afadin interacts with profilin, ${ }^{34}$ which is upregulated in rat Thy- 1 nephritis. ${ }^{31,33}$ Profilin activates monomeric actin units for subsequent polymerization steps at barbed ends of actin filaments and participates in the cortical actin assembly. Thus, afadin could modulate the actin modeling proximal to adhesion complexes through interaction with profilin. ${ }^{34}$ The imbalance in expression between profilin and afadin in proliferative nephritis may result in the disruption of links between the junctional complexes and cytoskeleton. Second, changes in afadin expression may modulate growth factor signaling. Glomerular reconstitution after a mesangial cell injury is controlled by several signaling pathways, and growth factors such as connective tissue growth factor, PDGF, and TGF $\beta 1$, which stimulate mesangial cell migration through the integrated dissolution of focal adhesion complexes and activation of cell polarization. ${ }^{35,36}$ These growth factors induce rapid phosphorylation of Akt/protein kinase $\mathrm{B}^{35,37}$ Afadin was recently identified as an Akt substrate, and its phosphorylation of Ser1718 perturbs cell adhesion and enhances migration. ${ }^{38}$ Loss of afadin expression may result in the modulation of polarized migration via alteration of the PI3-kinase/Akt signaling pathway. Finally, afadin at the mesangial cell-cell junction may affect the function of 
$\beta$-catenin. In mesangial cells, $\mathrm{Wnt} / \beta$-catenin signaling is implicated in mesangial survival and apoptosis. ${ }^{39-41}$ Although there is no evidence that afadin functions as a transcriptional coactivator, recent studies have implicated distinct roles for nuclear and cytoplasmic afadin. ${ }^{38,42}$ Further studies are required to fully elucidate the role of the afadin- $\beta$-catenin complex formation in intracellular or nuclear signaling.

Directed cell migration is an integrated process that is essential for embryonic development and has a key role in wound healing. Migration begins with cellular responses to an external signal, such as growth factors or the ECM, which elicits the extension of polarized protrusions in the direction of movement. ${ }^{43}$ During migration, the microtubule organizing center and Golgi apparatus reorient between the leading edge and the nucleus to direct and maintain migration. ${ }^{44}$ Although cell polarity proteins crucial for tight junction formation regulate directional migration in epithelial cells, ${ }^{45,46}$ the function of afadin in directional migration is context dependent. Afadin knockout in mice results in embryonic lethality due to disorganization of the ectoderm, impaired migration of the mesoderm, and impaired gastrulation. ${ }^{17,47}$ Indeed, afadin depletion in human colonic epithelial cells or NIH3T3 cells decreases the rate of cell migration. ${ }^{24,48}$ Afadin expression and its binding to Rap1 is required for the formation of the protein complex at the leading edge during directional cell movement. ${ }^{24}$ In contrast, migration is increased by afadin knockdown in breast carcinoma cells, ${ }^{49}$ and the loss of afadin expression in tumors correlates with larger tumors and poor metastasis-free survival in patients. ${ }^{50}$ These differences might arise from distinct cytoskeletal structures or adhesive properties of each cell type. In mesangial cells, afadin depletion resulted in the defective reorientation of the Golgi complex at the forward edge of the healing wound and delayed directional cell migration. The mesangial interposition observed in proliferative glomerulonephritis is characterized by the misdirected enhancement of cytoplasmic extensibility of mesangial cells. ${ }^{51}$ Therefore, downregulation of afadin in mesangial cells is likely to promote random, non-coordinated migration in activated mesangial cells, possibly leading to disruption of the glomerular structure.

Our results shed light on the possible role of changes in cell-cell adhesion in the aberrant front-rear polarity in mesangial cells in proliferative glomerulonephritis. Elucidation of polarized structures of mesangial cells in glomeruli and their regulatory mechanisms may improve our understanding of pathogenic responses to injury in glomeruli.

Supplementary Information accompanies the paper on the Laboratory Investigation website (http://www.laboratoryinvestigation.org)

\section{ACKNOWLEDGMENTS}

We express our gratitude to Professor $Y$ Takai, Kobe University, for providing plasmids. We appreciate Dr K Ishizuka, Tokyo Women's Medical University, for supporting the confocal microscopy experiments. We express our thanks to Ms R Onai for technical assistance. This work was supported in part by a
Grant-in-Aid for Scientific Research (B) (22390259 to YH and KM) and Grant-in-Aid for Scientific Research (C) (25461617 to HT, YH, HK, and MH) from the Ministry of Education, Culture, Sports, Science, and Technology of Japan.

\section{DISCLOSURE/CONFLICT OF INTEREST}

The authors declare no conflict of interest.

1. Kriz W, Elger M, Lemley K et al. Structure of the glomerular mesangium: a biomechanical interpretation. Kidney Int Suppl 1990;30:S2-S9.

2. Yaoita $\mathrm{E}$, Sato $\mathrm{N}$, Yoshida $\mathrm{Y}$ et al. Cadherin and catenin staining in podocytes in development and puromycin aminonucleoside nephrosis. Nephrol Dial Transplant 2002;17(Suppl 9):16-19.

3. Nameta M, Yaoita $\mathrm{E}$, Kato $\mathrm{N}$ et al. Mesangial cells connected by the $\mathrm{N}$-cadherin-catenin system in the rat kidney. Nephron Exp Nephrol 2009;112:e92-e98.

4. Yao J, Morioka T, Oite T. PDGF regulates gap junction communication and connexin43 phosphorylation by PI 3-kinase in mesangial cells. Kidney Int 2000;57:1915-1926.

5. Morioka T, Okada S, Nameta $M$ et al. Glomerular expression of connexin 40 and connexin 43 in rat experimental glomerulonephritis. Clin Exp Nephrol 2013;17:191-204.

6. Yamamoto T, Wilson CB. Quantitative and qualitative studies of antibody-induced mesangial cell damage in the rat. Kidney Int 1987;32:514-525.

7. Yamamoto T, Wilson CB. Complement dependence of antibodyinduced mesangial cell injury in the rat. J Immunol 1987;138: 3758-3765.

8. Hugo C, Shankland SJ, Bowen-Pope DF et al. Extraglomerular origin of the mesangial cell after injury. A new role of the juxtaglomerular apparatus. J Clin Invest 1997;100:786-794.

9. Daniel C, Albrecht $\mathrm{H}$, Ludke A et al. Nestin expression in repopulating mesangial cells promotes their proliferation. Lab Invest 2008;88: 387-397.

10. Daniel C, Ludke A, Wagner A et al. Transgelin is a marker of repopulating mesangial cells after injury and promotes their proliferation and migration. Lab Invest 2012;92:812-826.

11. lida $H$, Seifert $R$, Alpers $C E$ et al. Platelet-derived growth factor (PDGF) and PDGF receptor are induced in mesangial proliferative nephritis in the rat. Proc Natl Acad Sci USA 1991;88:6560-6564.

12. Floege J, Eitner F, Alpers CE. A new look at platelet-derived growth factor in renal disease. J Am Soc Nephrol 2008;19:12-23.

13. Waldherr R, Noronha IL, Niemir Z et al. Expression of cytokines and growth factors in human glomerulonephritides. Pediatr Nephrol 1993;7:471-478.

14. Schena FP, Gesualdo L, Montinaro V. Immunopathological aspects of immunoglobulin A nephropathy and other mesangial proliferative glomerulonephritides. J Am Soc Nephrol 1992;2(10 Suppl):S167-S172.

15. Mandai $\mathrm{K}$, Nakanishi $\mathrm{H}$, Satoh A et al. Afadin: A novel actin filamentbinding protein with one PDZ domain localized at cadherin-based cellto-cell adherens junction. J Cell Biol 1997;139:517-528.

16. Takai Y, Ikeda W, Ogita $\mathrm{H}$ et al. The immunoglobulin-like cell adhesion molecule nectin and its associated protein afadin. Annu Rev Cell Dev Biol 2008;24:309-342.

17. Takai $Y$, Nakanishi $H$. Nectin and afadin: novel organizers of intercellular junctions. J Cell Sci 2003;116(Pt 1):17-27.

18. Yamamoto T, Harada N, Kano K et al. The Ras target AF-6 interacts with ZO-1 and serves as a peripheral component of tight junctions in epithelial cells. J Cell Biol 1997;139:785-795.

19. Ooshio T, Kobayashi R, Ikeda W et al. Involvement of the interaction of afadin with ZO-1 in the formation of tight junctions in Madin-Darby canine kidney cells. J Biol Chem 2010;285:5003-5012.

20. Miyoshi J, Takai Y. Nectin and nectin-like molecules: biology and pathology. Am J Nephrol 2007;27:590-604.

21. Nakata S, Fujita N, Kitagawa $Y$ et al. Regulation of platelet-derived growth factor receptor activation by afadin through SHP-2: implications for cellular morphology. J Biol Chem 2007;282:37815-37825.

22. Shinosaki T, Notoya M, Nomura $Y$ et al. Glomerular epithelial cell injury accelerates the progression of antibody-induced mesangial proliferative nephritis. Exp Nephrol 2002;10:245-258.

23. Nobes CD, Hall A. Rho GTPases control polarity, protrusion, and adhesion during cell movement. J Cell Biol 1999;144:1235-1244. 
24. Miyata $\mathrm{M}$, Ogita $\mathrm{H}$, Komura $\mathrm{H}$ et al. Localization of nectin-free afadin at the leading edge and its involvement in directional cell movement induced by platelet-derived growth factor. J Cell Sci 2009;122(Pt 23): 4319-4329.

25. Kupfer A, Louvard D, Singer SJ. Polarization of the Golgi apparatus and the microtubule-organizing center in cultured fibroblasts at the edge of an experimental wound. Proc Natl Acad Sci USA 1982;79. 2603-2607.

26. Cortes $\mathrm{P}$, Mendez M, Riser BL et al. F-actin fiber distribution in glomerular cells: structural and functional implications. Kidney Int 2000;58:2452-2461.

27. Samanta D, Almo SC. Nectin family of cell-adhesion molecules: structural and molecular aspects of function and specificity. Cell Mol Life Sci 2014;72:645-658.

28. Takai $Y$, Miyoshi J, Ikeda $W$ et al. Nectins and nectin-like molecules: roles in contact inhibition of cell movement and proliferation. Nat Rev Mol Cell Biol 2008;9:603-615.

29. Johnson RJ, lida H, Alpers CE et al. Expression of smooth muscle cell phenotype by rat mesangial cells in immune complex nephritis. Alphasmooth muscle actin is a marker of mesangial cell proliferation. J Clin Invest 1991;87:847-858.

30. Tsurumi H, Harita $\mathrm{Y}$, Kurihara $\mathrm{H}$ et al. Epithelial protein lost in neoplasm modulates platelet-derived growth factor-mediated adhesion and motility of mesangial cells. Kidney Int 2014;86:548-557.

31. Tamura M, Tanaka H, Yashiro A et al. Expression of profilin, an actinbinding protein, in rat experimental glomerulonephritis and its upregulation by basic fibroblast growth factor in cultured rat mesangial cells. J Am Soc Nephrol 2000;11:423-433.

32. Clarkson MR, Murphy M, Gupta $S$ et al. High glucose-altered gene expression in mesangial cells. Actin-regulatory protein gene expression is triggered by oxidative stress and cytoskeletal disassembly. J Biol Chem 2002;277:9707-9712.

33. Peitsch WK, Hofmann I, Endlich $\mathrm{N}$ et al. Cell biological and biochemical characterization of drebrin complexes in mesangial cells and podocytes of renal glomeruli. J Am Soc Nephrol 2003;14: 1452-1463.

34. Boettner B, Govek EE, Cross J et al. The junctional multidomain protein AF- 6 is a binding partner of the Rap1A GTPase and associates with the actin cytoskeletal regulator profilin. Proc Natl Acad Sci USA 2000;97: 9064-9069.

35. Crean JK, Furlong $F$, Finlay $D$ et al. Connective tissue growth factor [CTGF]/CCN2 stimulates mesangial cell migration through integrated dissolution of focal adhesion complexes and activation of cell polarization. FASEB J 2004;18:1541-1543.

36. Kagami S, Kondo S, Loster $\mathrm{K}$ et al. Alpha1beta1 integrin-mediated collagen matrix remodeling by rat mesangial cells is differentially regulated by transforming growth factor-beta and platelet-derived growth factor-BB. J Am Soc Nephrol 1999;10:779-789.

37. Crean JK, Finlay D, Murphy M et al. The role of p42/44 MAPK and protein kinase $B$ in connective tissue growth factor induced extracellular matrix protein production, cell migration, and actin cytoskeletal rearrangement in human mesangial cells. J Biol Chem 2002;277:44187-44194.
38. Elloul S, Kedrin D, Knoblauch NW et al. The adherens junction protein afadin is an AKT substrate that regulates breast cancer cell migration. Mol Cancer Res 2014;12:464-476.

39. Bienz M. beta-Catenin: a pivot between cell adhesion and Wnt signalling. Curr Biol 2005;15:R64-R67.

40. Lin CL, Wang JY, Ko JY et al. Superoxide destabilization of beta-catenin augments apoptosis of high-glucose-stressed mesangial cells. Endocrinology 2008;149:2934-2942.

41. Lin CL, Wang JY, Huang YT et al.. Wnt/beta-catenin signaling modulates survival of high glucose-stressed mesangial cells. J Am Soc Nephrol 2006;17:2812-2820.

42. VanLeeuwen JE, Rafalovich I, Sellers K et al. Coordinated nuclear and synaptic shuttling of afadin promotes spine plasticity and histone modifications. J Biol Chem 2014;289:10831-10842

43. Raftopoulou M, Hall A. Cell migration: Rho GTPases lead the way. Dev Biol 2004;265:23-32.

44. Gomes ER, Jani S, Gundersen GG. Nuclear movement regulated by Cdc42, MRCK, myosin, and actin flow establishes MTOC polarization in migrating cells. Cell 2005;121:451-463.

45. Du D, Xu F, Yu L et al. The tight junction protein, occludin, regulates the directional migration of epithelial cells. Dev Cell 2010;18:52-63.

46. Suzuki A, Ohno S. The PAR-aPKC system: lessons in polarity. J Cell Sci 2006;119(Pt 6):979-987.

47. Ikeda W, Nakanishi $\mathrm{H}$, Miyoshi J et al. Afadin: A key molecule essential for structural organization of cell-cell junctions of polarized epithelia during embryogenesis. J Cell Biol 1999;146:1117-1132.

48. Severson EA, Lee WY, Capaldo CT et al. Junctional adhesion molecule $A$ interacts with Afadin and PDZ-GEF2 to activate Rap1A, regulate beta1 integrin levels, and enhance cell migration. Mol Biol Cell 2009;20: 1916-1925.

49. Fournier G, Cabaud O, Josselin E et al. Loss of AF6/afadin, a marker of poor outcome in breast cancer, induces cell migration, invasiveness and tumor growth. Oncogene 2011;30:3862-3874.

50. Letessier A, Garrido-Urbani S, Ginestier C et al. Correlated break at PARK2/FRA6E and loss of AF-6/Afadin protein expression areassociated with poor outcome in breast cancer. Oncogene 2007;26:298-307.

51. Kubo S, Kim ST, Takasugi $M$ et al. Pathogenetic mechanisms involved inmesangial interposition in IgA nephropathy. Nephron 1994;68:308-313.

(c) (i) (a) This work is licensed under a Creative Commons Attribution-NonCommercial-ShareAlike $\quad \mathbf{4 . 0}$ International License. The images or other third party material in this article are included in the article's Creative Commons license, unless indicated otherwise in the credit line; if the material is not included under the Creative Commons license, users will need to obtain permission from the license holder to reproduce the material. To view a copy of this license, visit http://creativecommons.org/licenses/by-nc$\mathrm{sa} / 4.0 /$ 